\title{
Force-Induced Reversal of -Eliminations: Stressed Disulfide Bonds in Alkaline Solution
}

Przemyslaw Dopieralski, ${ }^{*}$ Jordi Ribas-Arino,* Padmesh Anjukandi, Martin Krupicka, and Dominik Marx*

Dr. P. Dopieralski, Dr. J. Ribas-Arino, Dr. P. Anjukandi, Dr. M. Krupicka, Prof. Dr. D. Marx Lehrstuhl für Theoretische Chemie, Ruhr-Universität Bochum 44780 Bochum (Germany) E-mail:

przemyslaw.dopieralski@theochem.rub.de

jribasjr@yahoo.es

dominik.marx@theochem.rub.de

Dr. P. Dopieralski

Permanent address: Faculty of Chemistry, University of Wroclaw Joliot-Curie 14, 50-383 Wroclaw (Poland)

Dr. J. Ribas-Arino

Permanent address: Departament de Química Física and IQTCUB Universitat de Barcelona

Av. Diagonal 645, 08028 Barcelona (Spain)

Dr. M. Krupicka

Current address: Max-Planck-Institut für Chemische Energiekonversion

Stiftstrasse 34-36, 45470 Mülheim an der Ruhr (Germany) 


\begin{abstract}
Understanding the impact of tensile forces on disulfide bond cleavage is not only crucial to the breaking of cross-linkers in vulcanized materials such as strained rubber, but also to the regulation of protein activity by disulfide switches. By using ab initio simulations in the condensed phase, we investigated the response of disulfide cleavage by $\beta$-elimination to mechanical stress. We reveal that the ratedetermining first step of the thermal reaction, which is the abstraction of the $\beta$ proton, is insensitive to external forces. However, forces larger than about $1 \mathrm{nN}$ were found to reshape the free-energy landscape of the reaction so dramatically that a second channel is created, where the order of the reaction steps is reversed, turning $\beta$-deprotonation into a barrier-free follow-up process to $\mathrm{C}-\mathrm{S}$ cleavage. This transforms a slow and force-independent process with second-order kinetics into a unimolecular reaction that is greatly accelerated by mechanical forces.
\end{abstract}


The impact of tensile forces on the kinetics and reaction mechanisms of disulfide bond reductions has been intensely investigated in recent years. ${ }^{1-15}$ Much of the current interest in biochemistry stems from the increasingly acknowledged key role played by the reduction of so-called "disulfide switches" , commonly found in protein regions of pronounced mechanical strain, in the regulation of the activity of many proteins. ${ }^{14-18}$ Orthogonal to this function in living organisms is the force induced breaking of sulfur - based cross - linkers in vulcanized rubber, which is essential not only in waste rubber recycling (an urgent economic and environmental task faced by industry worldwide) ${ }^{19-21}$ but also in defining the mechanical fatigue properties of rubber. ${ }^{22,23}$

In landmark single - molecule experiments in the context of the covalent mechanochemistry ${ }^{24-28}$ of disulfide bond breaking, Fernandez and co-workers probed the cleavage of such bonds in aqueous alkaline solutions as a function of applied tensile force. ${ }^{3}$ These force-clamp atomic force microscopy (AFM) experiments revealed a "mechanochemical switch" at a force of about $0.5 \mathrm{nN}$ above which the accelerating effect of tensile force on the reaction rate was considerably diminished. ${ }^{3}$ In a recent computational study, ${ }^{12}$ we showed that the reaction probed in these experiments is $\mathrm{S}-\mathrm{S}$ bond cleavage triggered by a nucleophilic attack of an $\mathrm{OH}^{-}$ion onto one of the sulfur atoms by an $\mathrm{S}_{\mathrm{N}} 2$ mechanism. Upon studying the response of this reaction pathway to tensile stress, we disclosed that the mechanochemical switch originates from a force - induced conformational rearrangement of the disulfide bridge, which drives the disulfide into a conformation that is shielded against nucleophilic attack. Our subsequent work compared the force response of the $\mathrm{S}_{\mathrm{N}} 2$ mechanism, which is preferred at low mechanical stress, to other scenarios becoming relevant in the high-force regime. ${ }^{29}$

When it comes to disulfide bond cleavage reactions, it is well known that under certain circumstances, ${ }^{30-32}$ disulfides can undergo $\beta$-elimination in alkaline solution. This requires investigations into the response of this more complex reaction pathway to mechanical stress to achieve a detailed understanding of the mechanochemistry of disulfide bridges. Herein, based on ab initio molecular 
dynamics studies in the condensed phase enhanced with metadynamics sampling, ${ }^{33}$ we explore how the free - energy landscape associated with the $\beta$ elimination channel gets transformed under the action of external tensile stress. Unexpectedly, we disclose significant force-induced changes in the mechanism at intermediate forces, which reveals a novel reaction channel aside from the slow and force-insensitive bimolecular process in terms of a greatly accelerated unimolecular process.

The model system introduced here to explore the mechanical response of the $\beta$ elimination scenario greatly exceeds our previously studied minimal diethyl disulfide model in complexity ${ }^{12,29}$ (Figure 1, inset), and the model compound was hence solvated in a spacious bulk water periodic supercell that also hosts the attacking fully solvated $\mathrm{OH}^{-}$(aq). In particular, the disulfide model employed herein exhibits one of its sulfur atoms linked to a large organic moiety in which the $\beta$ - carbon atom of the disulfide is bonded to two fragments excised from the protein backbone used in the AFM experiments. ${ }^{3}$ This ensures that the acidity of the $\beta$-proton (see Figure 1, inset) is properly represented, whereas the far distant sulfur site is saturated using a methyl group. Two so-called collective variables (CVs) were introduced to span the free - energy landscape that characterizes $\beta$ - elimination (see the Supporting Information for details): The first one is the coordination number, $\mathrm{CN}_{\mathrm{C}-\mathrm{S}}$, between the carbon and sulfur atoms of the $\mathrm{C}-\mathrm{S}$ bond (denoted by CV1 in Figure 2). CV2 is the difference in the coordination number between the $\beta$-hydrogen and the carbon atom to which it is linked in the disulfide molecule, $\mathrm{CN}_{\mathrm{C}-\mathrm{H} \beta}$, and the coordination number of the $\beta$-hydrogen atom with respect to the oxygen atom of the hydroxide ion, that is, $\mathrm{CN}_{\mathrm{C}-\mathrm{H} \beta}-\mathrm{CN}_{\mathrm{C}-\mathrm{H} \beta-\mathrm{OH}-}$ 


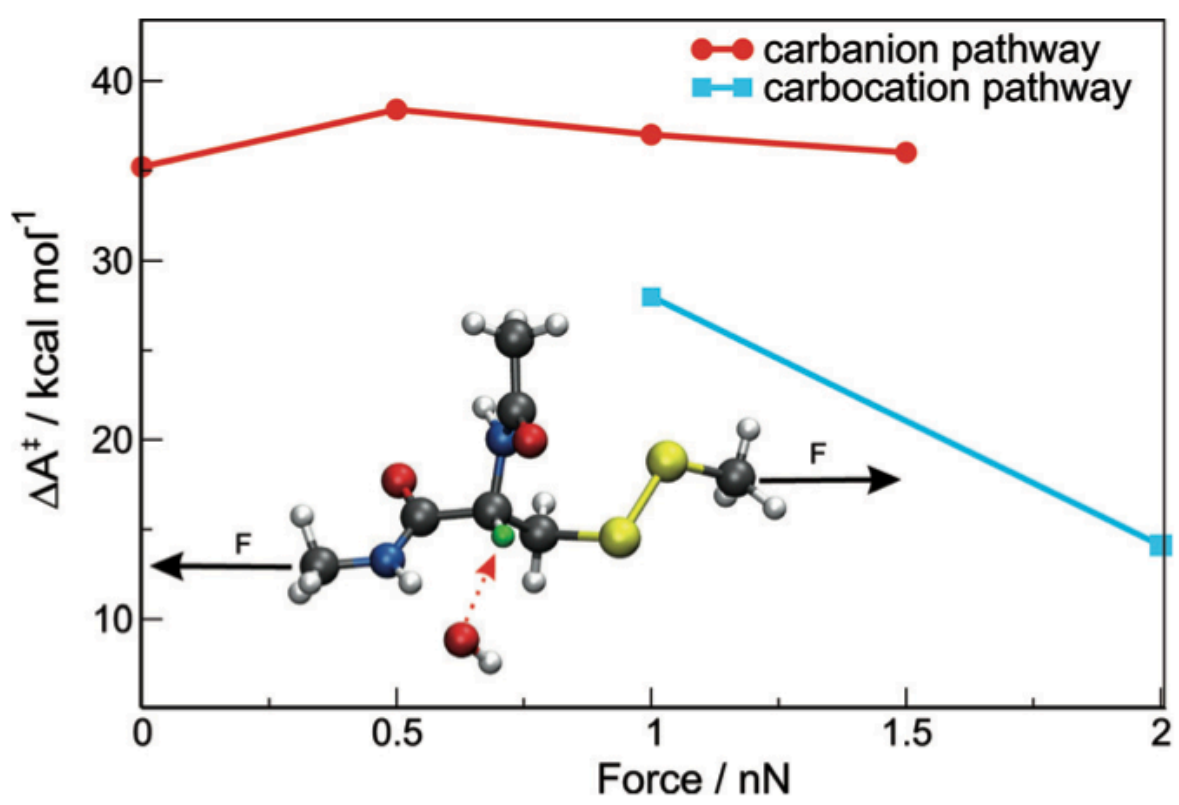

Figure 1. Force dependence of the activation free energy for $\beta$-elimination of the disulfide bond (see inset), corresponding to the rate-determining bimolecular and unimolecular first step of the reaction according to the carbanion $(\bullet)$ and carbocation $(\cdot)$ pathways, which are preferred at low and high force, respectively. The inset shows the large disulfide model currently employed together with the attacking hydroxide ion (dashed red arrow); all water molecules were removed for clarity. The $\beta$-hydrogen atom (green) gets abstracted as a proton by $\mathrm{OH}^{-}(\mathrm{aq})$. $\mathrm{C}$ black, $\mathrm{H}$ gray, $\mathrm{N}$ blue, $\mathrm{O}$ red, $\mathrm{S}$ yellow. Arrows indicate the $\mathrm{C}$ atoms on which constant mechanical forces of magnitude $F$ are exerted collinearly along a fixed direction in space.

The free energy surface (FES) obtained for $\beta$-elimination in the thermal limit (i.e., in the absence of any external mechanical force) demonstrates that this reaction follows a two - step mechanism (see Figure 2 A). In the rate-determining first step (which connects the reactant R to product P1 via transition state TS1), the $\mathrm{OH}^{-}$ion removes the $\beta$-proton of the disulfide by overcoming a significant free-energy barrier of $\Delta \mathrm{A} \neq \approx 35 \mathrm{kcal} \mathrm{mol}-1$ (see Figure 1 at $\mathrm{F}=0 \mathrm{nN}$ ). The resulting product $\mathrm{P} 1$ is a short-lived carbanion intermediate where the attacking $\mathrm{OH}^{-}$species, which has been turned into a water molecule by $\mathrm{H}$ abstraction, creates an $\mathrm{H}$-bonded bridge that connects one of the sulfur atoms with an oxygen atom of the amide bond (see Figure $2 \mathrm{~A}$, inset, P1). The second step $(\mathrm{P} 1 \rightarrow \mathrm{TS} 2 \rightarrow \mathrm{P} 2$ in Figure $2 \mathrm{~A}$ ) consists of simple $\mathrm{C}-\mathrm{S}$ bond cleavage with a vanishingly small and force-independent barrier on the order of $1 \mathrm{kcal} \mathrm{mol}^{-1}$; the thereby generated disulfide anion eventually accepts a proton from water. Overall, this is a two - step "irreversible carbanion" mechanism, ${ }^{34,35}(\mathrm{E} 1 \mathrm{cB}) \mathrm{I}$, that is characterized by second - order kinetics, where 
the first bimolecular step-involving the conjugate base and yielding carbanion P1-is rate - determining and essentially irreversible given that the second step is very fast. Indeed, this finding is in agreement with general expectations for the purely thermal reaction as electron - withdrawing groups in the $\beta$-position, such as the CONH2 group in our case, shift $\beta$-eliminations towards the E1cB mechanism. ${ }^{35}$ Moreover, strong bases and high base concentrations also favor $\mathrm{E} 1 \mathrm{cB},{ }^{35}$ which is certainly the case for our aqueous alkaline solution.

A
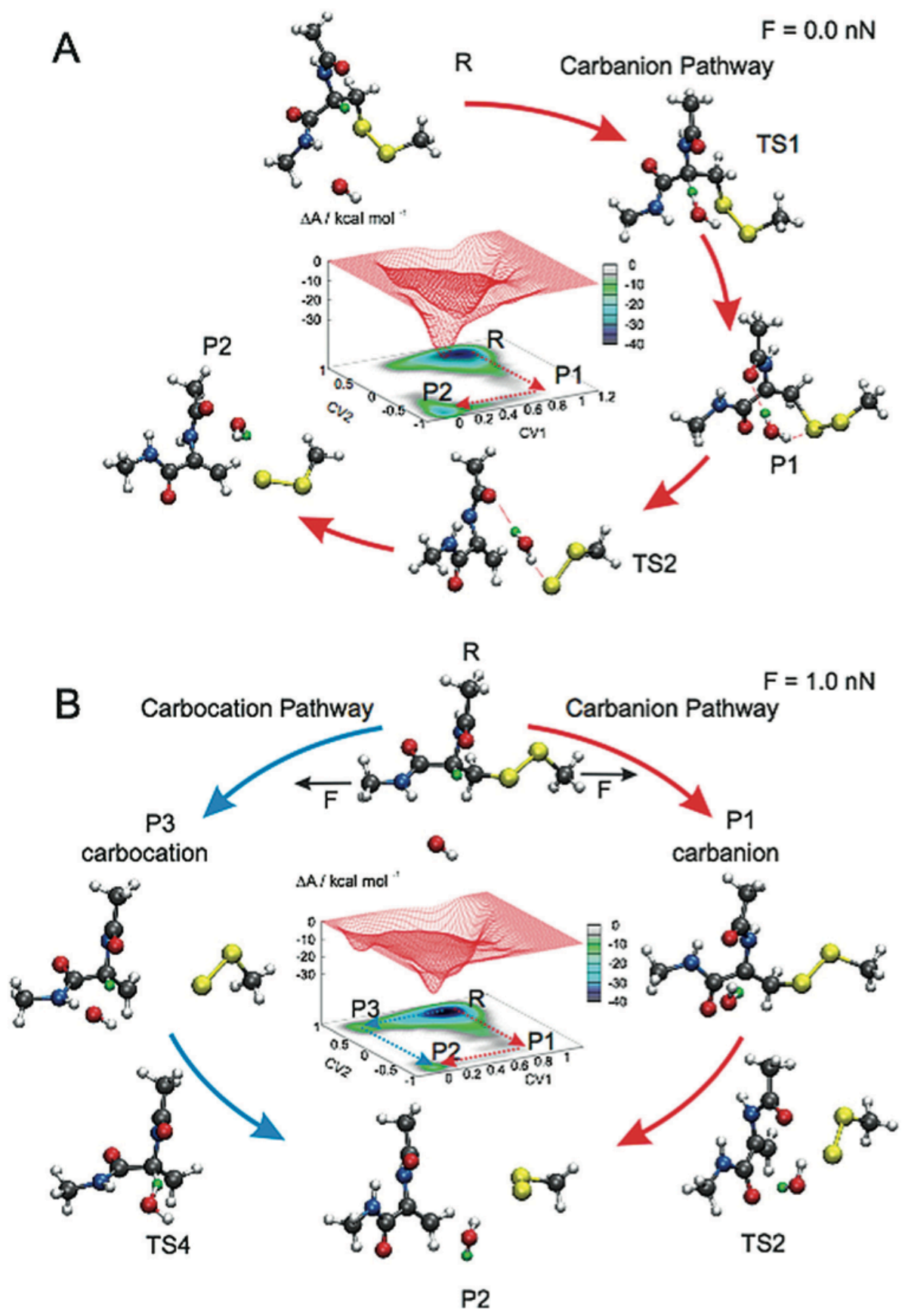

Figure 2. A) Free-energy landscape for $\beta$-elimination (see Figure 1 ) in the thermal limit (i.e., at $\mathrm{F}=0 \mathrm{nN}$ ) where only the carbanion pathway is operating. B) Force-transformed 
free-energy landscape for $\beta$-elimination (see Figure 1) at a constant force of $\mathrm{F}=1 \mathrm{nN}$ where the carbocation channel is energetically preferred over the still operating carbanion pathway. Red and blue arrows indicate the carbanion and carbocation pathways, respectively, whereas $R, P_{n}$, and $T_{n}$ denote reactants, intermediates/products, and transition states, respectively, for which representative snapshots sampled from ab initio metadynamics are shown.

Having successfully deciphered the thermal $\beta$-elimination reaction mechanism, we focused on the effect of finite mechanical tensile forces on this process. This investigation was performed by means of isotensional metadynamics sampling 28,36 carried out in the presence of different, but constant external forces (see Figure 1). For each fixed value of the external force, the isotensional metadynamics simulations lead to a different so - called "force - transformed FES",36 which provides the proper reaction landscape including the mechanical distortion. ${ }^{28}$

At a force of $0.5 \mathrm{nN}$, the entire mechanism remains unchanged when compared to the purely thermally activated process (see the corresponding force-transformed FES in the Supporting Information, Figure S3). Interestingly, however, the barrier $\Delta \mathrm{A} \neq$ for the rate-determining first step remains roughly constant according to Figure 1, which means that the external force does not result in any acceleration of the overall reaction. Larger forces do not lead to a reduction of this free-energy barrier either (Figure 1). Our simulations thus clearly reveal that the ratedetermining step of the $(\mathrm{E} 1 \mathrm{cB})_{\mathrm{I}}$ process, that is, $\beta$-proton abstraction, is fully decoupled from the applied mechanical force. Other examples of force-insensitive chemical processes whose reaction coordinates are orthogonal to the mechanical coordinates can be found in Refs. 5 and 37-39.

A dramatic change of scenario is observed upon reaching an intermediate tensile force of $1 \mathrm{nN}$. Indeed, Figure $2 \mathrm{~B}$ discloses that the force-transformed FES enables a different reaction channel: First, the carbocation P3 is obtained by cleaving the $\mathrm{C}-\mathrm{S}$ bond without involvement of the base, $\mathrm{OH}^{-}(\mathrm{aq})$, which only subsequently abstracts the $\beta$-proton $(\mathrm{R} \rightarrow \mathrm{P} 3 \rightarrow \mathrm{P} 2)$; the disulfide anion generated after the first step eventually stabilizes itself by abstracting a proton from the solvent. The activation free energy of the rate - determining first step at $\mathrm{F}=1.0 \mathrm{nN}, \Delta \mathrm{A} \neq \approx 28 \mathrm{kcal} \mathrm{mol}^{-1}$ according to Figure 1, is much smaller than that associated with the rate - 
determining first step of the $(\mathrm{E} 1 \mathrm{cB})_{\mathrm{I}}$ process, which is $37 \mathrm{kcal} \mathrm{mol}^{-1}$ for $\beta-\mathrm{H}$ abstraction by $\mathrm{OH}^{-}(\mathrm{aq})$ at $1 \mathrm{nN}$. However, the generation of carbocations in aqueous solution appears to be an utmost unrealistic scenario. Indeed, our simulations show that no carbocations are generated in the limit of the thermally activated reaction (i.e., at $\mathrm{F}=0 \mathrm{nN}$, see Figure $2 \mathrm{~A}$ ), and that their generation is also not feasible at moderate forces below approximately $1 \mathrm{nN}$ (see Figure 1). So, why is carbocation generation enabled by sufficiently large mechanical forces?

Mechanistic analysis of the novel scenario shows that the same final product P2 as before is obtained, yet by a vastly different mechanism. It was classified to be a two-step E1 process ${ }^{35}$ as the rate-determining first step leads, in a unimolecular process $(\mathrm{R} \rightarrow \mathrm{P} 3)$, to a carbocation that readily loses the $\beta$-proton (see $\mathrm{P} 3 \rightarrow \mathrm{P} 2$ in Figure 2 B), therefore leading to first - order kinetics. The picture underlying this scenario is that the internal strain generated by mechanically stretching the molecule is so large that the covalent bond is simply ripped apart $(\mathrm{R} \rightarrow \mathrm{P} 3)$ before any other process occurs. The nascent carbocation is short - lived and stabilizes itself quickly after its generation by detaching the $\beta$-proton into the basic solution. This view is supported by the observation that the rate - determining $\mathrm{R} \rightarrow$ P3 process, and thus the entire E1 pathway, is very responsive to tensile forces: The activation free energy, $\Delta \mathrm{A} \neq$, is reduced to half from about $28 \mathrm{kcal} \mathrm{mol}^{-1}$ at 1 $\mathrm{nN}$ to approximately $14 \mathrm{kcal} \mathrm{mol}^{-1}$ at $2 \mathrm{nN}$. Moreover, Mulliken population analysis (see Figure S1) reveals that the $\alpha$-carbon atom involved in the $\mathrm{C}-\mathrm{S}$ bond cleavage of P3 intermittently acquires a positive partial charge. Last but not least, reference - coupled cluster calculations of $\mathrm{C}-\mathrm{S}$ bond breaking using a reduced model embedded in continuum water provide no support for a homolytic diradical mechanism instead of the heterolytic ionic pathway involving the reported carbocationic intermediate. In conclusion, the E1 reaction channel favored at sufficiently large forces not only implies a significant acceleration of a hitherto force - insensitive reaction, but also a force - induced change from a bimolecular to a unimolecular process and from second-order to first-order reaction kinetics. 
Given the vastly different reaction channel that is preferred at forces beyond $1 \mathrm{nN}$, our final analysis addresses the nature of the elementary $\beta$-proton abstraction step. Figure 3 nicely reveals that C-S cleavage (dark - brown line) occurs after/before $\beta-\mathrm{H}$ abstraction by $\mathrm{OH}^{-}$(green lines) in the carbanion/carbocation mechanism depicted in panels $\mathrm{B} / \mathrm{D}$ at $\mathrm{F}=1.0 \mathrm{nN}$. The $\mathrm{OH}^{-}$(aq) hydration shell (red line) is more flexible in the carbanion scenario, whereas mostly two water molecules remain hydrogen - bonded along the carbocation pathway (see panels A/C, respectively). Moreover, the $\beta$-proton-abstracting $\mathrm{OH}^{-}$(aq) forms a stable hydrogen bond to one of the NH groups of the reactant molecule (blue line). The fact that this $\mathrm{H}$-bond is broken shortly after the $\beta$-proton is abstracted, but largely locked before, suggests that this hydrogen bond keeps the nucleophile at the reaction center. Indeed, such an hydrogen - bonded intermediate state (HBIS) has been observed for base - catalyzed peptide hydrolysis. ${ }^{38}$ Here, only a slight migration of $\mathrm{OH}^{-}$from such an HBIS configuration to the $\mathrm{C}-\mathrm{H} \beta$ group is required to achieve the elementary elimination step.
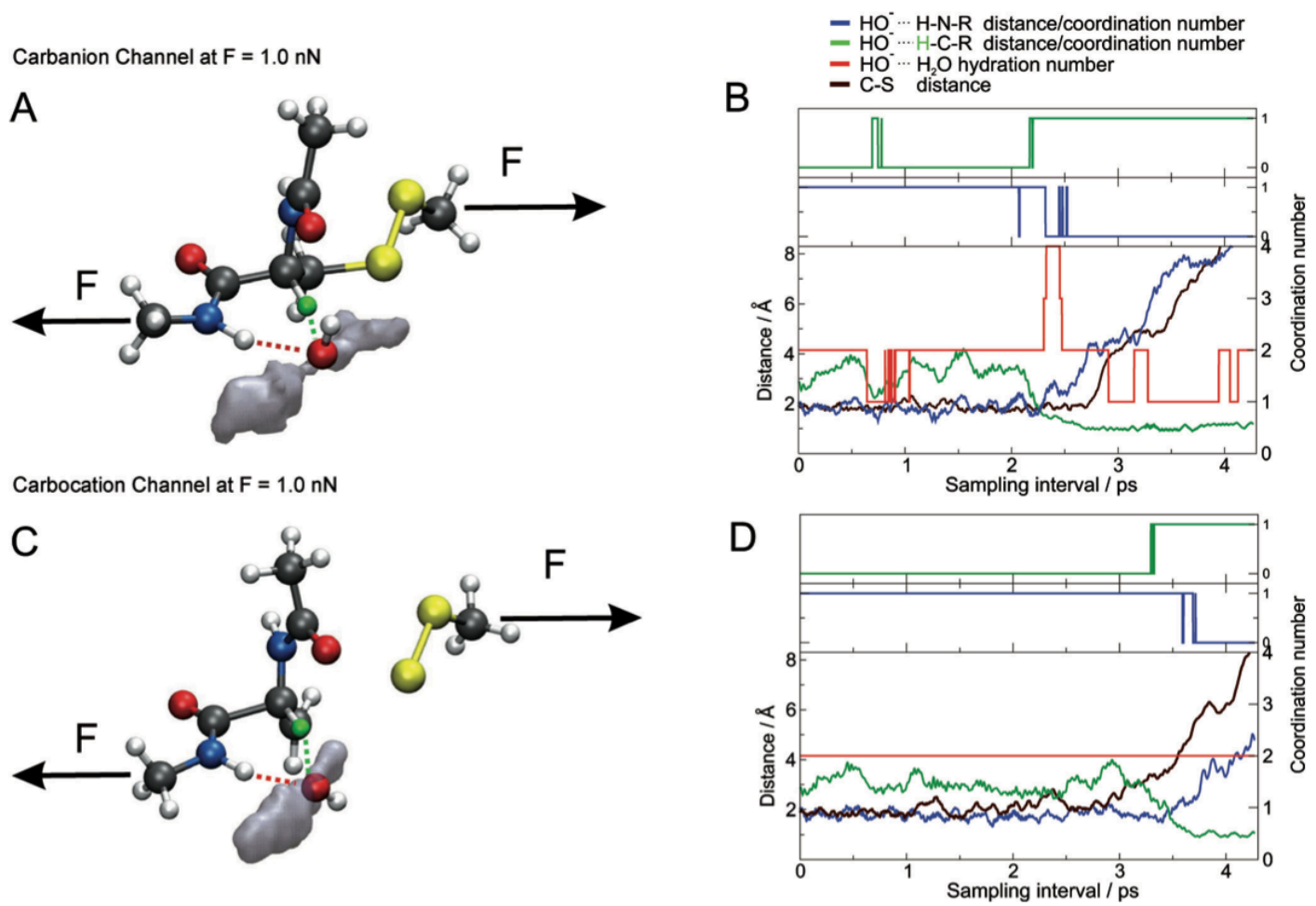

Figure 3. Elementary elimination step involving b-proton abstraction by $\mathrm{OH}^{-}(\mathrm{aq})$ at a constant force of $\mathrm{F}=1 \mathrm{nN}$ along the carbanion and carbocation reaction channels (top and bottom, respectively). The left panels depict configuration snapshots of the reactive complex when $\mathrm{OH}^{-}(\mathrm{aq})$ is about to abstract the b-proton (green dotted line) and to break 
its stabilizing hydrogen bond to $\mathrm{H}-\mathrm{N}$-(red dotted line) together with a contour plot of the corresponding spatial distribution functions of the oxygen site of $\mathrm{OH}$ in the $\mathrm{N}-\mathrm{H} \cdots \mathrm{Hb}$ plane sampled shortly before, during, and after $\mathrm{Hb}$ abstraction and thus $\mathrm{H}_{2} \mathrm{O}$ formation. The bulk water environment present in the simulations is not shown. The panels on the right show in their lower parts the evolution of the following distances along the two pathways: dark brown: C-S distance; blue and green lines: distance from the $\mathrm{O}$ site of $\mathrm{OH}^{-}$to the $\mathrm{H}$ sites of the $\mathrm{H}-\mathrm{N}$ - and $\mathrm{H}_{\beta}-\mathrm{C}$ - groups, respectively; red line: number of water molecules hydrogenbonded to $\mathrm{OH}^{-}(\mathrm{aq})$. The top parts show the coordination of $\mathrm{OH}^{-}(\mathrm{aq})$ with respect to the $\mathrm{H}$ sites of the $\mathrm{H}-\mathrm{N}$ - and $\mathrm{H}_{\beta}-\mathrm{C}$ - groups using again blue and green lines, where the final jumps to zero and unity correspond to the termination of the HBIS (see text) and to $\beta$-proton abstraction, respectively; a simple distance criterion of $\mathrm{R}<2.4 \AA$ was used to visualize the hydration and coordination numbers. Note that $\mathrm{OH}^{-}$(aq) changes to $\mathrm{H}_{2} \mathrm{O}$ (aq) along the red line shortly before the green and blue lines cross.

\section{Acknowledgements}

We are grateful to partial financial support from the Deutsche Forschungsgemeinschaft (Reinhart Koselleck Grant "Understanding Mechanochemistry", MA 1547/9) and Cluster of Excellence "RESOLV", EXC 1069)), the Alexander von Humboldt Stiftung (Humboldt Fellowship to J.R.-A.), and the Spanish Government (Ramón y Cajal Fellowship to J.R.-A.), as well as by the National Science Center Poland (2014/13/B/ST4/05009), and the Ministry of Science and Higher Education Poland (627/STYP/9/2014) for fellowships to P.D. We also gratefully acknowledge the Gauss Centre for Supercomputing (GCS) for providing computing time for a GCS Large Scale Project on JUQUEEN at the Jülich Supercomputing Centre (JSC) as well as additional computational support by HPC-RESOLV, BOVILAB@RUB, the Rechnerverbund NRW, the Wroclaw Supercomputer Center (WCSS), the GaleraACTION Cluster, and the Academic Computer Center in Gdańsk (CI TASK). 


\section{References}

[1] A. P. Wiita, S. R. K. Ainavarapu, H. H. Huang, J. M. Fernandez, Proc. Natl. Acad. Sci. USA 2006, 103, $7222-7227$.

[2] A. P. Wiita, R. Perez-Jimenez, K. A. Walther, F. Gräter, B. J. Berne, A. Holmgren, J.M. Sanchez-Ruiz, J.M. Fernandez, Nature 2007, 450, 124 - 127.

[3] S. Garcia-Manyes, J. Liang, R. Szoszkiewicz, T. L. Kuo, J. M. Fernandez, Nat. Chem. 2009, 1, $236-242$.

[4] J. Liang, J. M. Fernandez, ACS Nano 2009, 3, 1628 - 1645.

[5] T. J. Kucharski, Z. Huang, Q. Z. Yang, Y. Tian, N. C. Rubin, C. D. Concepcion, R. Boulatov, Angew. Chem. Int. Ed. 2009, 48,

7040 - 7043 ; Angew. Chem. 2009, 121, 7174 - 7177.

[6] W. Li, F. Gräter, J. Am. Chem. Soc. 2010, 132, 16790-16795.

[7] M.F.Iozzi, T.Helgaker, E.Uggerud, J.Phys.Chem.A2011,115,2308 - 2315.

[8] S. Keten, C.-C. Chou, A. C. van Duin, M. J. Buehler, J. Mech. Behav. Biomed. Mater. 2012, 5, $32-40$.

[9] F. Hofbauer, I. Frank, Chem. Eur. J. 2012, 18, 16332 - 16338.

[10] I. Popa, P. Kosuri, J. Alegre-Cebollada, S. Garcia-Manyes, J. M. Fernandez, Nat. Protoc. 2013, 8, $1261-1276$.

[11] Y. Tian, T. J. Kucharski, Q. Z. Yang, R. Boulatov, Nat. Commun. 2013, 4, 2538.

[12] P. Dopieralski, J. Ribas-Arino, P. Anjukandi, M. Krupicka, J. Kiss, D. Marx, Nat. Chem. 2013, 5, $685-691$.

[13] Y. Li, A. Nese, K. Matyjaszewski, S. S. Sheiko, Macromolecules, 2013, 46, 7196 7201.

[14] P. Anjukandi, P. Dopieralski, J. Ribas-Arino, D. Marx, PLoS One 2014, 9, e108812.

[15] B. Zhou, I. B. Baldus, W. Li, S. A. Edwards, F. Gräter, Biophys. J. 2014, 107, 672 -681 .

[16] P. J. Hogg, Trends Biochem. Sci. 2003, 28, 210 - 214.

[17] J. Ahamed, H. H. Versteeg, M. Kerver, V. M. Chen, B. M. Mueller, P. J. Hogg, W.

Ruf, Proc. Natl. Acad. Sci. USA 2006, 103, 13932- 13937.

[18] M. A. Wouters, S. W. Fan, N. L. Haworth, Antioxid. Redox Signaling 2010, 12, 53 $-91$. 
[19] X. Guo, D. Xiang, G. Duan, P. Mou, Waste Manage. 2010, 30, 4 -10.

[20] X. Zhang, Z. Lu, D. Tian, H. Li, C. Lu, J. Appl. Polym. Sci. 2013, 127, 4006 - 4014.

[21] H. Rust, US8957119 B2, 2015.

[22] W. V. Mars, A. Fatemi, Rubber Chem. Technol. 2004, 77, 391 -412.

[23] B. N. J. Persson, O. Albohr, G. Heinrich, H. Ueba, J. Phys. Condens. Matter 2005, 17, R1071 - R1142.

[24] M. K. Beyer, H. Clausen-Schaumann, Chem. Rev. 2005, 105, 2921 - 2948.

[25] M. M. Caruso, D. A. Davis, Q. Shen, S. A. Odom, N. R. Sottos, S. R. White, J. S. Moore, Chem. Rev. 2009, 109, 5755 - 5798.

[26] A. L. Black, J. M. Lenhardt, S. L. Craig, J. Mater. Chem. 2011, 21, 1655- 1663.

[27] Z. Huang, R. Boulatov, Chem. Soc. Rev. 2011, 40, 2359- 2384.

[28] J. Ribas-Arino, D. Marx, Chem. Rev. 2012, 112, 5412- 5487.

[29] P. Dopieralski, J. Ribas-Arino, P. Anjukandi, M. Krupicka, D. Marx, unpublished results.

[30] M. Friedman, J. Agric. Food Chem. 1999, 47, 1295- 1319.

[31] S. Cohen, C. Price, J. Vlasak, J. Am. Chem. Soc. 2007, 129, 6976- 6977.

[32] B. Lagrain, K. de Vleeschouwer, I. Rombouts, K. Brijs, M. Hendrickx, J. Delcour, J. Agric. Food Chem. 2010, 58, 10761- 10767.

[33] D. Marx, J. Hutter, Ab Initio Molecular Dynamics: Basic Theory and Advanced Methods, Cambridge University Press, Cambridge, 2009.

[34] F. G. Bordwell, Acc. Chem. Res. 1972, 5, 374- 381.

[35] M. B. Smith, March's Advanced Organic Chemistry: Reactions, Mechanisms and Structure, 7th ed., Wiley, Hoboken, 2013.

[36] P. Dopieralski, J. Ribas-Arino, D. Marx, Angew. Chem. Int. Ed. 2011, 50, 71057108;

[37] J. Ribas-Arino, M. Shiga, D. Marx, Chem. Eur. J. 2009, 15, 13331- 13335.

[38] F. Xia, A. Baranowska, S. Cheng, F. Gräter, J. Phys. Chem. B 2011, 115, 1012610132.

[39] R. Groote, B. M. Szyja, F. A. Leibfarth, C. J. Hawker, N. L. Doltsinis, R. P. Sijbesma, Macromolecules 2014, 47, 1187- 1192. 